\title{
TORSION POINTS ON THETA DIVISORS AND SEMIHOMOGENEOUS VECTOR BUNDLES
}

\author{
GIUSEPPE PARESCHI
}

\begin{abstract}
We generalize to $n$-torsion a result of Kempf's describing 2-torsion points lying on a theta divisor. This is accomplished by means of certain semihomogeneous vector bundles introduced and studied by Mukai and Oprea. As an application, we prove a sharp upper bound for the number of $n$-torsion points lying on a theta divisor and show that this is achieved only in the case of products of elliptic curves, settling in the affirmative a conjecture of Auffarth, Pirola and Salvati Manni.
\end{abstract}

\section{INTRODUCTION}

Let $(A, \underline{\theta})$ be a complex $g$-dimensional principally polarized abelian variety. This paper is concerned with the set of $n$-torsion points lying on the theta divisors, where $n$ is any fixed integer $\geq 2$.

We choose once for all a symmetric divisor $\Theta$ representing the polarization. For $x \in A$ we denote by $t_{x}: A \rightarrow A$ the translation by $x$, and $\Theta_{x}$ the effective divisor corresponding to the line bundle $t_{x}^{*} \mathcal{O}_{A}(\Theta)$ (i.e. $\left.\Theta_{x}=\Theta-x\right)$. We set

$$
\Theta_{x}(n):=\# A[n] \cap \Theta_{x}
$$

where $A[n]$ is the group of all $n$-torsion points of $A$.

A result of Kempf ([8, Theorem 3]) asserts that, for $x, y \in A$ the corank of the multiplication map of global sections

$$
H^{0}\left(A, t_{x}^{*} \mathcal{O}_{A}(2 \Theta)\right) \otimes H^{0}\left(A, t_{y}^{*} \mathcal{O}_{A}(2 \Theta)\right) \longrightarrow H^{0}\left(A, t_{x}^{*} \mathcal{O}_{A}(2 \Theta) \otimes t_{y}^{*} \mathcal{O}_{A}(2 \Theta)\right)
$$

coincides with the number $\Theta_{y-x}(2)$ (we refer to [18, §2] for the translation into the present setting of Kempf's statement, which contains a slight mistake). Our first result is an extension of Kempf's theorem to $n$-torsion points, for arbitrary $n$. This is achieved using certain semihomogeneous vector bundles, denoted $\mathrm{W}_{a, b}$, introduced and systematically studied by Oprea in [14] (as a consequence of Mukai's theory of semihomogeneous vector bundles, [11]). When $a$ and $b$ are coprime positive integers, the $\mathrm{W}_{a, b}$ 's are defined as simple, semihomogeneous and symmetric vector bundles such that

$$
\operatorname{rkW}_{a, b}=a^{g} \quad \text { and } \quad \operatorname{det} \mathrm{W}_{a, b}=\mathcal{O}_{A}(\Theta)^{a^{g-1} b} .
$$

If $a$ is odd there is a unique such vector bundle, while if $a$ is even they are not unique when $g \geq 2$. We refer to the next section for generalities on such vector bundles. Our generalization of Kempf's theorem (which is recovered for $a=b=1$ ) is the following

The author was partially supported by the MIUR Department of Excellence Project awarded to the Department of Mathematics, University of Rome Tor Vergata, CUP E83C18000100006. 
Theorem A. Let $a$ and $b$ be coprime positive integers. Let $\mathrm{W}_{a, a+b}$ and $\mathrm{W}_{b, a+b}$ be two vector bundles as above. For $x, y \in A$ the number $\Theta_{y-x}(a+b)$ is equal to the corank of the multiplication map of global sections

$$
m_{a, b}(x, y): H^{0}\left(A, t_{x}^{*} \mathrm{~W}_{a, a+b}\right) \otimes H^{0}\left(A, t_{y}^{*} \mathrm{~W}_{b, a+b}\right) \longrightarrow H^{0}\left(A, t_{x}^{*} \mathrm{~W}_{a, a+b} \otimes t_{y}^{*} \mathrm{~W}_{b, a+b}\right)
$$

(As it is easy to check, the source and target of the above map have the same dimension, namely $(a+b)^{2 g}$.) Note that if $a$ or $b$ are even, say $a$, the above map $m_{a, b}$ depends on a choice of a vector bundle $\mathrm{W}_{a, a+b}$, but we will neglect this in the notation. A special role will be played by the particular case

$$
m_{1, n-1}(x, y): H^{0}\left(A, t_{x}^{*} \mathcal{O}_{A}(n \Theta)\right) \otimes H^{0}\left(A, t_{y}^{*} \mathrm{~W}_{n-1, n}\right) \longrightarrow H^{0}\left(A, t_{x}^{*} \mathcal{O}_{A}(n \Theta) \otimes t_{y}^{*} \mathrm{~W}_{n-1, n}\right)
$$

obtained for $a=1$ and $n:=a+b$.

In view of Theorem $\mathrm{A}$, it is useful to consider criteria for the surjectivity of the multiplication of global sections of semihomogeneous vector bundles, in analogy with well known classical theorems for line bundles (due to Mumford, Koizumi, Kempf and others, see e.g. [4, §7.2] [7, §6.2], [6, §8]). In fact an optimal result in this direction was already proved years ago by Popa and the author ([17, Theorem 7.30]). We restate it more expressively as Theorem 4.1 below. In turn this is an ingredient of the proof of the following lower bound for the rank of the multiplication maps appearing in Theorem $\mathrm{A}$

Theorem B. In the notation of Theorem $\left[\right.$, $\quad r k\left(m_{a, b}(x, y)\right) \geq\left((a+b)^{2}-1\right)^{g}$ for all $x, y \in A$.

The last result of this note, in fact our original motivation, is the proof of the following conjecture of Auffarth, Pirola and Salvati Manni on the maximal number of $n$-torsion points on a theta divisor (see [2]). The case $n=2$, which was conjectured earlier by Marcucci and Pirola ([10]), was proved by Salvati Manni and the author in [18].

Theorem C. For all $x \in A$

$$
\Theta_{x}(n) \leq n^{2 g}-\left(n^{2}-1\right)^{g} .
$$

Moreover equality holds if and only if $A$ is a product of elliptic curves and $\mathcal{O}_{A}\left(\Theta_{x}\right)=\bigotimes_{i} \mathcal{O}_{E_{i}}\left(z_{i}\right)$ where $z_{i}$ are $n$-torsion points.

Note that the upper bound of the statement is just the combination of Theorems $\mathrm{A}$ and $\mathrm{B}$, The remaining part is proved in Section 5 .

It would be interesting to have effective results along these lines for irreducible principal polarizations. To this purpose it should be kept in mind that, thanks to a recent result of Auffarth and Codogni ([1]), there are irreducible theta divisors containing abelian subvarieties of dimension up to $g-2$, hence containing at least $n^{2(g-2)} n$-torsion points for all $n$. On the other hand, by Raynaud's theorem on the Manin-Mumford conjecture, the overall number of torsion points contained in a theta divisor is finite unless it contains translates of positive-dimensional abelian subvarieties by torsion points.

Acklowledgements. The author thanks: Dragos Oprea for pointing out a gap in an earlier draft of this paper, Mihnea Popa for many conversations about semihomogeneous vector bundles a long time ago, Riccardo Salvati Manni for his encouragement and many discussions and suggestions, and the referee for very accurate remarks. 


\section{Preliminaries on the Vector Bundles $\mathrm{W}_{a, b}$}

Here we recall some basic facts about the vector bundles $\mathrm{W}_{a, b}$ introduced by Oprea in [14. Let $(A, \underline{\theta})$ be a $g$-dimensional p.p.a.v. and let $\Theta$ be a fixed symmetric theta divisor. For a pair of coprime positive integers $a$ and $b$ we consider simple semihomogeneous vector bundles $\mathrm{W}$ such that

$$
r k \mathrm{~W}=a^{g} \quad \operatorname{det} \mathrm{W}=\mathcal{O}_{A}(\Theta)^{a^{g-1} b} .
$$

Vector bundles with the above properties do exist and they are unique up to tensorization with an $a^{g}$-torsion line bundle ([11, Theorem 7.11 and Remark 7.13]). Denoting by $a_{A}: A \rightarrow A$ the isogeny $x \mapsto a x$, we have that

$$
a_{A}^{*} \mathrm{~W} \cong\left(\mathcal{O}_{A}(\Theta)^{a b}\right)^{\oplus a^{g}}
$$

(see [14, 2.3.1]). Moreover such W's satisfy the index theorem with index 0 (IT(0) for short), meaning that $h^{i}\left(\mathrm{~W}_{a, b} \otimes \alpha\right)=0$ for all $i>0$ and $\alpha \in \widehat{A}$ (we denote $\widehat{A}=\operatorname{Pic}^{0} A$ the dual abelian variety). Recalling that the degree of the isogeny $a_{A}$ is $a^{2 g}$, it follows that

$$
h^{0}(A, \mathrm{~W})=\chi(W)=a^{g}\left(\frac{b}{a}\right)^{g}=b^{g} .
$$

Another useful fact about the vector bundles satisfying the above condition (2.1) is that they are globally generated as soon as $b>a$. This follows from the criterion asserting that a vector bundle $E$ on $A$ is globally generated as soon as $E(-\Theta)$ is $\operatorname{IT}(0)$ ([15, Theorem 2.1]). Indeed (2.2) yields that $\mathrm{W}(-\Theta)$ is $\operatorname{IT}(0)$ if and only if $b>a$.

For odd $a$, imposing the supplementary condition that $\mathrm{W}$ is symmetric, i.e. $(-1)_{A}^{*} \mathrm{~W} \cong \mathrm{W}$, it turns out that there is a unique such vector bundle up to isomorphism ([14, §2.1]). It is denoted $\mathrm{W}_{a, b}$. Also for even $a$ such symmetric vector bundles do exist (for example the dual of the FourierMukai transform of the vector bundle $\mathrm{W}_{b, a}$ ) but they are not unique for $g \geq 2$. For even $a$ Oprea defines a unique such vector bundle $\mathrm{W}_{a, b}$ by means of the Schrödinger representation ([14, $\S 2.1$ and (16)]). However this is not important for our purposes, since we will consider any simple symmetric semihomogeneous vector bundle $\mathrm{W}$ satisfying (2.1). We denote $\mathcal{W}_{a, b}$ the set of all isomorphism classes of such simple symmetric semihomogeneous vector bundles and a vector bundle $\mathrm{W} \in \mathcal{W}_{a, b}$ will be usually denoted $\mathrm{W}_{a, b}$ 西

We consider the subgroup

$$
\Sigma\left(\mathrm{W}_{a, b}\right)=\left\{\alpha \in \widehat{A} \mid \mathrm{W}_{a, b} \otimes \alpha \cong \mathrm{W}_{a, b}\right\} .
$$

We have that, independently on the parity of $a$,

$$
\Sigma\left(\mathrm{W}_{a, b}\right)=\widehat{A}[a]
$$

( $a$-torsion line bundles, see [11, Corollary 7.2]).

Given $\mathrm{W}_{a, b} \in \mathcal{W}_{a, b}$, the other vector bundles, say $\mathrm{W}_{a, b}^{\prime}$ (possibly isomorphic to $\mathrm{W}_{a, b}$ ) whose isomorphism class lies in $\mathcal{W}_{a, b}$ are those of the form

$$
\mathrm{W}_{a, b}^{\prime} \cong \mathrm{W}_{a, b} \otimes \beta
$$

for $\beta \in \widehat{A}\left[a^{g}\right]$ such that $(-1)_{A}^{*}\left(\mathrm{~W}_{a, b} \otimes \beta\right) \cong \mathrm{W}_{a, b} \otimes \beta^{-1} \cong \mathrm{W}_{a, b} \otimes \beta$. Therefore $\beta^{2} \in \widehat{A}[a]$. This, together with the condition $\beta \in \widehat{A}\left[a^{g}\right]$ implies that, if $a$ is odd (or $g=1$ ) then $\beta \in \widehat{A}[a]=\Sigma\left(\mathrm{W}_{a, b}\right)$. Hence, as mentioned above, there is a unique such an isomorphism class ([14, §2.1]).

\footnotetext{
${ }^{1}$ Here our notation differs from the one of Oprea, as he denotes $\mathrm{W}_{a, b}$ the unique vector bundle in $\mathcal{W}_{a, b}$ defined, as mentioned above, via the Schrödinger representation.
} 
In the proof of Theorem $\AA$ the following (slight variant of a) result of Oprea will be in use. For $a$ and $b$ coprime positive integers one considers the isogeny

$$
\mu_{b, a}: A \times A \rightarrow A \times A \quad(z, t) \mapsto(b z+a t, z-t) .
$$

Proposition 2.1. (Oprea) Keeping the above notation and assumptions, given a pair of vector bundles $\left(\mathrm{W}_{a, a+b}, \mathrm{~W}_{b, a+b}\right) \in \mathcal{W}_{a, a+b} \times \mathcal{W}_{b, a+b}$ there is a vector bundle $W_{a b, 1} \in \mathcal{W}_{a b, 1}$ such that

$$
\mu_{b, a}^{*}\left(\mathrm{~W}_{a b, 1} \otimes \mathcal{O}_{A}(\Theta)\right) \cong \mathrm{W}_{a, a+b} \otimes \mathrm{W}_{b, a+b} .
$$

Proof. Let $a$ and $b$ be coprime positive integers. For $a$ and $b$ both odd (or $g=1$ and arbitrary $a$ and $b$, see [3]) all vector bundles appearing in the statement are unique and the Proposition is exactly Oprea's [14, Proposition 1]. Next, we assume that $g \geq 2$ and $a$ and $b$ are still coprime, but one of them, say $a$, is even. We fix a reference bundle $\overline{\mathrm{W}}_{a b, 1} \in \mathcal{W}_{a b, 1}$. Oprea's argument still proves that the determinant of $\mu_{b, a}^{*}\left(\overline{\mathrm{W}}_{a b, 1} \otimes \mathcal{O}_{A}(\Theta)\right)$ is equal to the one of $\mathrm{W}_{a, a+b} \otimes \mathrm{W}_{b, a+b}$, and that

$$
\mu_{b, a}^{*}\left(\overline{\mathrm{W}}_{a b, 1} \otimes \mathcal{O}_{A}(\Theta)\right) \cong\left(\mathrm{W}_{a, a+b} \otimes \delta\right) \otimes\left(\mathrm{W}_{b, a+b} \otimes \gamma\right)
$$

for suitable $(\delta, \gamma) \in \widehat{A}\left[a^{g}\right] \times \widehat{A}\left[b^{g}\right]$. We claim that, moreover, both the vector bundles $E:=\mathrm{W}_{a, a+b} \otimes \delta$ and $F:=\mathrm{W}_{b, a+b} \otimes \gamma$ are symmetric. Indeed an immediate computation shows that $\left(-1_{A}, 1_{A}\right) \circ$ $\mu_{b, a}=\left(1_{A},-1_{A}\right) \circ \mu_{b, a} \circ\left(-1_{A},-1_{A}\right)$. Therefore, since $\overline{\mathrm{W}}_{a b, 1}$ and $\mathcal{O}_{A}(\Theta)$ are both symmetric, pulling back $\overline{\mathrm{W}}_{a b, 1} \otimes \mathcal{O}_{A}(\Theta)$ under the morphism $\left(-1_{A}, 1_{A}\right) \circ \mu_{b, a}$ we get that $\left(-1_{A}, 1_{A}\right)^{*}(E \otimes F) \cong$ $\left(1_{A},-1_{A}\right)^{*}(E \otimes F)$. This proves what was claimed. Hence, since $b$ is odd, $\mathrm{W}_{b, a+b} \cong \mathrm{W}_{b, a+b} \otimes \gamma$, i.e. $\gamma \in \widehat{A}[b]$. Moreover $\delta^{2} \in \Sigma\left(\mathrm{W}_{a, a+b}\right)=\widehat{A}[a]$.

To conclude the proof, we show that we can replace $\overline{\mathrm{W}}_{a b, 1}$ with another vector bundle in $\mathrm{W}_{a b, 1} \in \mathcal{W}_{a b, 1}$ such that (2.5) is satisfied. For any $\alpha \in \widehat{A}$ we have that $\mu_{b, a}^{*}\left(\alpha \otimes \mathcal{O}_{A}\right)=\left(\alpha^{b}, \alpha^{a}\right)$. Therefore

$$
\mu_{b, a}^{*}\left(\left(\overline{\mathrm{W}}_{a b, 1} \otimes \alpha\right) \otimes \mathcal{O}_{A}(\Theta)\right) \cong\left(\mathrm{W}_{a, a+b} \otimes \delta \otimes \alpha^{b}\right) \otimes\left(\mathrm{W}_{b, a+b} \otimes \gamma \otimes \alpha^{a}\right) .
$$

Taking any $\alpha$ such that $\alpha^{b}=\delta^{-1}$ we have that $\alpha^{2} \in \widehat{A}[a b]$ (hence also $\alpha \in \widehat{A}\left[(a b)^{g}\right]$ as soon as $g>1$ ). Therefore $\overline{\mathrm{W}}_{a b, 1} \otimes \alpha \in \mathcal{W}_{a b, 1}$. As above, by uniqueness when $b$ is odd, we have that $\mathrm{W}_{b, a+b} \cong \mathrm{W}_{b, a+b} \otimes \gamma \otimes \alpha^{a}$. Hence

$$
\mu_{b, a}^{*}\left(\left(\overline{\mathrm{W}}_{a b, 1} \otimes \alpha\right) \otimes \mathcal{O}_{A}(\Theta)\right) \cong \mathrm{W}_{a, a+b} \otimes \mathrm{W}_{b, a+b} .
$$

\section{Proof of Theorem A}

We essentially follow Kempf's argument, with some simplifications. Let

$$
\left(\mathrm{W}_{a, a+b}, \mathrm{~W}_{b, a+b}\right) \in \mathcal{W}_{a, a+b} \times \mathcal{W}_{b, a+b} .
$$

To render the argument more transparent we first prove the result for $(x, y)=(0,0)$.

The multiplication map $m_{a, b}(0,0)$ is the map $H^{0}\left(r_{\Delta}\right)$, where $r_{\Delta}$ is the restriction to the diagonal

$$
r_{\Delta}: \mathrm{W}_{a, a+b} \otimes \mathrm{W}_{b, a+b} \rightarrow\left(\mathrm{W}_{a, a+b} \otimes \mathrm{W}_{b, a+b}\right)_{\mid \Delta} .
$$

We apply Proposition 2.1, ensuring that

$$
\mu_{b, a}^{*}\left(\left(\mathrm{~W}_{a b, 1}\right) \otimes \mathcal{O}_{A}(\Theta)\right) \cong \mathrm{W}_{a, a+b} \otimes \mathrm{W}_{b, a+b} .
$$

Moreover, since $\mu_{b, a}^{*}\left(\mathcal{O}_{A \times\{0\}}\right)=\mathcal{O}_{\Delta}$, it follows that $r_{\Delta}=\mu_{b, a}^{*}(\rho)$, where $\rho$ is the restriction map

$$
\rho: \mathrm{W}_{a b, 1} \otimes \mathcal{O}_{A}(\Theta) \rightarrow\left(\mathrm{W}_{a b, 1} \otimes \mathcal{O}_{A}(\Theta)\right)_{\mid A \times\{0\}} .
$$


(By the way we note that, since the restricted map $\left(\mu_{b, a}\right)_{\mid \Delta}: \Delta \rightarrow A \times\{0\}$ is identified to the isogeny $(a+b)_{A}: A \rightarrow A$, it follows that $\left.\mathrm{W}_{a, a+b} \otimes \mathrm{W}_{b, a+b} \cong(a+b)_{A}^{*} \mathrm{~W}_{a b, 1}\right)$.

The kernel of the isogeny $\mu_{b, a}$ is $\Delta[a+b]:=\{(z, z) \mid(a+b) z=0\}$. Therefore, since

$$
r_{\Delta}=\mu_{b, a}^{*}(\rho),
$$

the multiplication map $m_{a, b}(0,0)=H^{0}\left(r_{\Delta}\right)$ decomposes as

$$
\bigoplus_{\alpha \in \widehat{A}[a+b]}\left(H^{0}\left(A \times A,\left(\mathrm{~W}_{a b, 1} \otimes P_{\alpha}\right) \otimes\left(\mathcal{O}_{A}(\Theta) \otimes P_{\alpha}\right)\right) \rightarrow H^{0}\left(A \times A,\left(\left(\mathrm{~W}_{a b, 1} \otimes P_{\alpha}\right) \otimes\left(\mathcal{O}_{A}(\Theta) \otimes P_{\alpha}\right)\right)_{\mid A \times\{0\}}\right) .\right.
$$

Via the isomorphism induced by the principal polarization $A \rightarrow \widehat{A}$, the above can be written as

$$
\bigoplus_{z \in A[a+b]}\left(H^{0}\left(A \times A, t_{z}^{*} \mathrm{~W}_{a b, 1} \otimes t_{z}^{*} \mathcal{O}_{A}(\Theta)\right) \stackrel{\lambda_{z}}{\longrightarrow} H^{0}\left(A \times A,\left(t_{z}^{*} \mathrm{~W}_{a b, 1} \otimes t_{z}^{*} \mathcal{O}_{A}(\Theta)\right)_{\mid A \times\{0\}}\right)\right) .
$$

Notice that, by (2.3),$H^{0}\left(A, \mathrm{~W}_{a b, 1}\right)=1$, so that the individual maps $\lambda_{z}$ appearing above are maps of 1-dimensional vector spaces. Hence the assertion of the theorem follows from the fact that the scalar $\lambda_{z}$ vanishes if and only if $A \times\{0\} \subset A \times \Theta_{z}$, i.e. $z \in \Theta$.

In the general case the proof is similar. In the first place, applying $t_{-x}^{*}$ we can assume that $x=0$. Via translation on the second factor we identify the map $m_{a, b}(0, y)$ of the statement to the map

$$
H^{0}\left(A, \mathrm{~W}_{a, a+b}\right) \otimes H^{0}\left(A, \mathrm{~W}_{b, a+b}\right) \longrightarrow H^{0}\left(A, \mathrm{~W}_{a, a+b} \otimes t_{y}^{*} \mathrm{~W}_{b, a+b}\right) .
$$

This is the $H^{0}$ of the restriction map

$$
r_{\Delta_{y}}: \mathrm{W}_{a, a+b} \otimes \mathrm{W}_{b, a+b} \rightarrow\left(\mathrm{W}_{a, a+b} \otimes \mathrm{W}_{b, a+b}\right)_{\mid \Delta_{y}}
$$

where $\Delta_{y}=d^{-1}(y)$ (here $d$ is the difference map $\left.A \times A \rightarrow A,(z, t) \mapsto z-t\right)$. We have that $\mathcal{O}_{\Delta_{y}}=\mu_{b, a}^{*}\left(\mathcal{O}_{A \times\{y\}}\right)$. The rest of the proof is unchanged.

\section{Multiplication of global Sections of Semihomogeneous Vector Bundles}

A surjectivity criterion for multiplication maps. We recall [17, Theorem 7.30], mentioned in the Introduction. In the case of interest for this paper, namely semihomogeneous vector bundles whose first Chern class is a power of a principal polarization, it can be stated as follows. Following Mukai ([11]), for a vector bundle $E$ on $A$ we write

$$
\delta_{E}=\frac{c_{1}(E)}{\mathrm{rk}(E)} \in N S(A) \otimes \mathbb{Q} .
$$

If $c_{1}(E)$ is a multiple of $\underline{\theta}$ we denote also $\mu_{E}$ the rational number defined by

$$
\delta_{E}=\mu_{E} \underline{\theta} .
$$

Theorem 4.1. (Pareschi-Popa) Let $E$ and $F$ be semihomogeneous vector bundles on $A$ such that $c_{1}(E)$ and $c_{1}(F)$ are multiples of $\underline{\theta}$. If

$$
\mu_{F}>1 \quad \text { and } \quad \mu_{E}>\frac{\mu_{F}}{\mu_{F}-1}
$$

then the multiplication map of global sections

$$
H^{0}(A, E) \otimes H^{0}(A, F) \rightarrow H^{0}(A, E \otimes F)
$$

is surjective. 
Note that for line bundles one recovers the classical fact that the multiplication map of a second power and a third power of a line bundle representing $\underline{\theta}$ is surjective.

Here we show that Theorem 4.1] is just the restatement of [17, Theorem 7.30], asserting that the multiplication map as in the statement is surjective as soon as both $E(-\Theta)$ and $F(-\Theta)$ satisfy $\operatorname{IT}(0)$ and

$$
\delta_{E(-\Theta)}+\delta_{\widehat{\Phi}_{\mathcal{P}}(F(-\Theta))}>0
$$

where $\widehat{\Phi}_{\mathcal{P}}: D(A) \rightarrow D(\widehat{A})$ is the Fourier-Mukai transform associated to the Poincaré bundle. Let us explain how to get the statement of Theorem 4.1 from this. In the first place we recall that, for a semihomogeneous vector bundle $G$, the $\operatorname{IT}(0)$ condition is equivalent to $\delta_{G}>0$. This follows, for example, from [11, Lemma 6.11], stating that

$$
r_{A}^{*} G \cong(\operatorname{det} G)^{r} \otimes H
$$

where $r:=\operatorname{rk} G, r_{A}$ denotes, as usual, the isogeny $x \mapsto r x$, and $H$ is a homogeneous vector bundle (indeed a homogeneous vector bundle is a direct sum of vector bundles of the form $\mathrm{U} \otimes \alpha$, where $\alpha \in \widehat{A}$ and $\mathrm{U}$ is a unipotent vector bundle, namely a vector bundle having a filtration $0=\mathrm{U}_{0} \subset \mathrm{U}_{1} \subset \cdots \subset \mathrm{U}_{n-1} \subset \mathrm{U}_{n}=\mathrm{U}$, with $\mathrm{U}_{i} / U_{i-1} \cong \mathcal{O}_{A}$ for $i=1, \ldots, n$, see [11, Theorem 4.17]).

Using the formulas

$$
\delta_{F \otimes G}=\delta_{F}+\delta_{G} \quad \text { and } \quad \delta_{G^{\vee}}=-\delta_{G},
$$

it follows that the condition that $\mu_{F}-1>0$, i.e. the first hypothesis of Theorem 4.1, is equivalent to the fact that $F(-\Theta)$ satisfies $\operatorname{IT}(0)$.

If this is the case then, by base change, the complex $\widehat{\Phi}_{\mathcal{P}}(F(-\Theta))$ is a sheaf in cohomological degree 0 , in fact a locally free sheaf. Next, we recall that, for a semihomogenous vector bundle $G$ satisfying $\operatorname{IT}(0)$, the vector bundle $\widehat{\Phi}_{\mathcal{P}}(G)$ is again semihomogeneous (this follows from the fact that $\widehat{\Phi}_{\mathcal{P}}$ exchanges translation with tensorization with a line bundle in $\widehat{A}$, see [12, (3.1)]). Finally we claim that if $G$ is such that $c_{1}(G)$ is a multiple of $\underline{\theta}$ then also $c_{1}\left(\widehat{\Phi}_{\mathcal{P}}(G)\right)$ is a multiple of $\underline{\theta}$ and the following beautiful formula holds:

$$
\mu_{\widehat{\Phi}_{\mathcal{P}}(G)}=-\frac{1}{\mu_{G}} .
$$

This translates the hypothesis (4.2) into the numerical condition

$$
\mu_{E}-1-\frac{1}{\mu_{F}-1}>0
$$

i.e. the second inequality in the hypothesis of Theorem 4.1.

Finally, we briefly indicate the proof of (4.5). This is certainly well known to the experts but we couldn't find an explicit reference. We recall that, for $\lambda \in \mathbb{Q}, r_{A}^{*}(\lambda \underline{\theta})=r^{2} \lambda \underline{\theta}$. Therefore from (4.3) it follows that $c h(G)=r \exp \left(\mu_{G} \underline{\theta}\right)$. Then a well known calculation using GRR and the Fourier-Mukai transform at the level of Chow rings modulo numerical equivalence (see e.g. the proof of [14, Lemma 2]) shows that $\operatorname{ch}\left(\widehat{\Phi}_{\mathcal{P}}(G)\right)=r\left(\mu_{G}\right)^{g} \exp \left(-\mu_{G}^{-1} \underline{\theta}\right)$. Therefore $\delta_{\widehat{\Phi}_{\mathcal{P}}(G)}=-\mu_{G}^{-1} \underline{\theta}$.

Proof of Theorem B. By Theorem $\mathrm{A}$ for fixed $x, y \in A$ the maps $m_{a, b}(x, y)$ have the same rank for all $a, b$ (coprime) with $a+b=n$ (and for all representatives $\left.\left(\mathrm{W}_{a, a+b}, \mathrm{~W}_{b, a+b}\right) \in \mathcal{W}_{a, a+b} \times \mathcal{W}_{b, a+b}\right)$. Therefore it is enough to prove the statement for $(a, b)=(n-1,1)$. As in the proof of Theorem 
A. we can furthermore assume that $x=0$. Let us fix $y \in A$. For general $z \in A$ we consider the commutative diagram

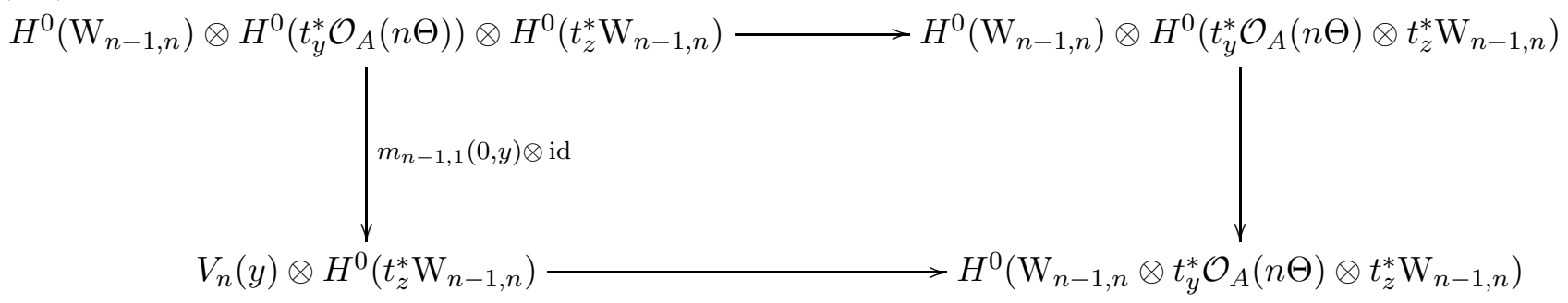

where $V_{n}(y)$ denotes the image of the map $m_{n-1,1}(0, y)$. By Theorem $\mathrm{A}$ the top horizontal map is surjective for general $z \in A$. By Theorem 4.1 the right vertical map is surjective for all $z \in A$ (here we use that $\mu_{\mathrm{W}_{a, b}}=\frac{b}{a}$ and (4.4) $)$. Therefore the bottom horizontal map is surjective for general $z \in A$. Hence

$$
\operatorname{dim} V_{n}(y) \geq \frac{\chi\left(\mathrm{W}_{n-1, n}^{\otimes 2} \otimes \mathcal{O}_{A}(n \Theta)\right)}{\chi\left(\mathrm{W}_{n-1, n}\right)} .
$$

By (2.3) we have that $\chi\left(\mathrm{W}_{n-1, n}\right)=n^{g}$. Using (2.2) one gets easily that $\chi\left(\mathrm{W}_{n-1, n}^{\otimes 2} \otimes \mathcal{O}_{A}(n \Theta)\right)=$ $(n-1)^{g} n^{g}(n+1)^{g}$. The result follows.

\section{Proof of Theorem C}

It is easy to check that the bound of Theorem $\mathrm{C}$ is attained by line bundles of the form $\mathcal{O}_{A}\left(\Theta_{x}\right)=\otimes_{i} \mathcal{O}_{E_{i}}\left(z_{i}\right)$ on products of elliptic curves $E_{i}$, where the $z_{i}$ 's are $n$-torsion points. Conversely, the second part of Theorem $\mathrm{C}$ asserts that this is the only case. To prove this, the main point consists in showing that if the bound is attained then $\Theta$ must be reducible and therefore the p.p.a.v. $(A, \underline{\theta})$ decomposes as a product of lower dimensional p.p.a.v's.

Proof for $\mathbf{g}>\mathbf{2}$. According to Theorem $\mathrm{A}$, what we need to show is that in the irreducible case the rank of the multiplication maps $m_{n-1, n}(0, y)$ is $>\left(n^{2}-1\right)^{2}$ for all $y$.

For $y \in A$ and $n>1$ let us consider the following divisor

$$
E_{y, n}:=\sum_{\eta \in A[n]} \Theta_{y+\eta} .
$$

An immediate consequence of Theorem $\mathrm{A}$ is the following (where, for reasons apparent in what follows, the notation $(x, y)$ in the statement of Theorem $\mathrm{A}$ has been switched to $(y, z))$ :

Corollary 5.1. Let $y \in A$. The map

$$
m_{a, b}(y, z): H^{0}\left(A, t_{y}^{*} W_{a, a+b}\right) \otimes H^{0}\left(A, t_{z}^{*} \mathrm{~W}_{b, a+b}\right) \longrightarrow H^{0}\left(A, t_{y}^{*} \mathrm{~W}_{a, a+b} \otimes t_{z}^{*} \mathrm{~W}_{b, a+b}\right)
$$

is singular if and only if $z \in \operatorname{Supp} E_{y, a+b}$.

In order to prove Theorem C] we see the map of Corollary 5.1 and all maps of diagram (4.6) as the fiberwise maps of maps of locally free sheaves. This is well known and it is done as follows. Following [15] (see also [16]), given two coherent sheaves $\mathcal{F}$ and $\mathcal{G}$, we define their (derived) skew Pontryagin product

$$
\mathcal{F} \hat{*} \mathcal{G}:=d_{*}(\mathcal{F} \otimes \mathcal{G})
$$

where $d: A \times A \rightarrow A$ is the difference map. We make the simplifying assumption that both sheaves $\mathcal{F}$ and $\mathcal{G}$ are locally free, semihomogeneous, and they satisfy IT(0) (these conditions will be always 
satisfied by the sheaves appearing in what follows). By [16, Proposition 2.9] the IT(0) condition for $\mathcal{F}$ and $\mathcal{G}$ implies that also the vector bundle $\mathcal{F} \otimes \mathcal{G}$ satisfies IT(0). In turn, this implies by base change that: $R^{i} d_{*}(\mathcal{F} \otimes \mathcal{G})=0$ for $i \neq 0, \mathcal{F} \hat{*} \mathcal{G}$ is a locally free sheaf (in degree 0 ), and

$$
d_{*}(F \otimes G) \otimes \mathbb{C}(z) \cong H^{0}\left(A \times A,(F \otimes G)_{\mid \Delta_{z}}\right)=H^{0}\left(A, \mathcal{F} \otimes t_{z}^{*} \mathcal{G}\right)
$$

for all $z \in A$ (see (3.2)). Thus the multiplication map of global sections

$$
H^{0}(A, \mathcal{F}) \otimes H^{0}\left(A, t_{z}^{*} \mathcal{G}\right) \rightarrow H^{0}\left(A, F \otimes t_{z}^{*} G\right)
$$

is naturally identified, via the isomorphism

$$
\operatorname{id} \otimes t_{z}^{*}: H^{0}(A, \mathcal{F}) \otimes H^{0}(A, \mathcal{G}) \rightarrow H^{0}(A, \mathcal{F}) \otimes H^{0}\left(A, t_{z}^{*} \mathcal{G}\right),
$$

to the fiber map at $z$ of the map of $\mathcal{O}_{A}$-modules:

$$
H^{0}(A, \mathcal{F}) \otimes H^{0}(A, \mathcal{G}) \otimes \mathcal{O}_{A} \cong d_{23 *}\left(\mathcal{F} \otimes \mathcal{O}_{A} \otimes \mathcal{G}\right) \rightarrow d_{23 *}\left(\left(\mathcal{F} \otimes \mathcal{O}_{A} \otimes \mathcal{G}\right)_{\mid \Delta_{12}}\right) \cong \mathcal{F} \hat{*} \mathcal{G}
$$

where $d_{23}\left(x_{1}, x_{2}, x_{3}\right)=\left(x_{2}-x_{3}\right)$ and $\Delta_{12}=\left\{\left(x_{1}, x_{2}, x_{3}\right) \mid x_{1}=x_{2}\right\}$. (Note that $d_{*}\left(\mathcal{O}_{A} \otimes \mathcal{G}\right)$ is trivial and canonically isomorphic to $H^{0}(A, \mathcal{G}) \otimes \mathcal{O}_{A}$, as is most easily seen via the automorphism of $A \times A,(x, y) \mapsto(x, x-y)$, sending $p_{2}$ to $d$ and leaving $p_{1}$ unchanged. Therefore $d_{23 *}\left(\mathcal{F} \nabla \mathcal{O}_{A} \otimes \mathcal{G}\right) \cong$ $\left.H^{0}(A, \mathcal{F}) \otimes H^{0}(A, \mathcal{G}) \otimes \mathcal{O}_{A}.\right)$ sections

More generally, given another $\operatorname{IT}(0)$ sheaf on $A$, say $\mathcal{H}$, the multiplication map of global

$$
H^{0}(A, \mathcal{F}) \otimes H^{0}\left(A, \mathcal{H} \otimes t_{z}^{*} \mathcal{G}\right) \rightarrow H^{0}\left(A, \mathcal{F} \otimes \mathcal{H} \otimes t_{z}^{*} \mathcal{G}\right)
$$

is naturally identified the fiber map at $z$ of the map of $\mathcal{O}_{A}$-modules:

$$
H^{0}(A, \mathcal{F}) \otimes(\mathcal{H} \hat{*} \mathcal{G}) \cong d_{23 *}(\mathcal{F} \otimes \mathcal{H} \otimes \mathcal{G}) \rightarrow d_{23 *}\left((\mathcal{F} \otimes \mathcal{H} \otimes \mathcal{G})_{\mid \Delta_{12}}\right) \cong(\mathcal{F} \otimes \mathcal{H}) \hat{*} \mathcal{G}
$$

After these preliminaries, we can proceed with the proof. Let us fix $y \in A$. Then diagram (4.6) is identified to the diagram of fiber maps at $z \in A$ of the commutative diagram of $\mathcal{O}_{A}$-modules, with surjective vertical maps,

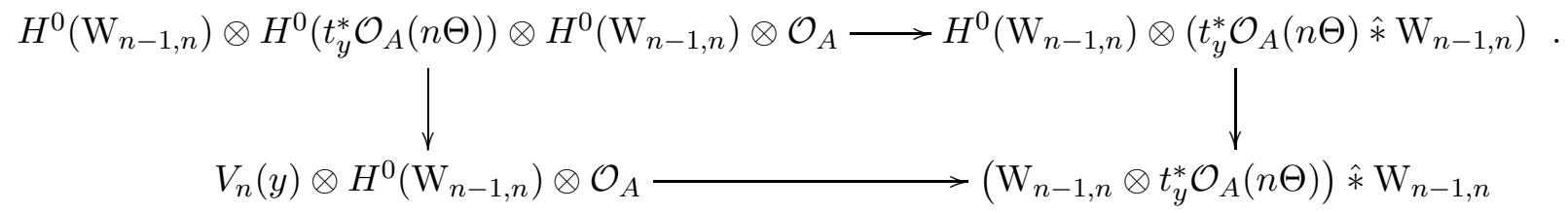

After routine calculations (summarized below), based on well known results of Mukai and Oprea, one computes

$$
c_{1}\left(\mathrm{~W}_{a, a+b} \hat{*} \mathrm{~W}_{b, a+b}\right)=(a+b)^{2 g} \underline{\theta}
$$

and

$$
c_{1}\left(\left(\mathrm{~W}_{a, a+b} \otimes \mathrm{W}_{b, a+b}\right) \hat{*} \mathrm{~W}_{a, a+b}\right)=(a+b)^{g+2} a^{g-1}(a+2 b)^{g-1} \underline{\theta} .
$$

In particular, for $a=n-1$ and $b=1$ one gets

$$
c_{1}\left(\mathrm{~W}_{n-1, n} \hat{*} \mathcal{O}_{A}(n \Theta)\right)=n^{2 g} \underline{\theta}
$$

and

$$
c_{1}\left(\left(\mathrm{~W}_{n-1, n} \otimes \mathcal{O}_{A}(n \Theta)\right) \hat{*} \mathrm{~W}_{n-1, n}\right)=n^{g+2}(n-1)^{g-1}(n+1)^{g-1} \underline{\theta} .
$$


Assume that $\Theta$ is irreducible, and, as above, let us fix $y \in A$. From Corollary [5.1] and (5.6) it follows that the effective divisor defined by the vanishing of the determinant of the map

$$
H^{0}\left(t_{y}^{*} \mathcal{O}_{A}(n \Theta)\right) \otimes H^{0}\left(\mathrm{~W}_{n-1, n}\right) \otimes \mathcal{O}_{A} \rightarrow\left(t_{y}^{*} \mathcal{O}_{A}(n \Theta)\right) \hat{*} \mathrm{~W}_{n-1, n}
$$

is the divisor $E_{y, n}$ of (5.1) (note that if $\Theta$ is irreducible then $E_{y, n}$ has no multiple components). Now assume that $\operatorname{dim} V_{n}(y)=(n)^{2 g}-\left((n)^{2}-1\right)^{g}$, which corresponds precisely to the bound in Theorem C. Then, as shown in the previous section, the source and target of the bottom horizontal map of (5.3) have the same rank. The determinant of this map vanishes on an effective divisor $D_{y, n}$, which is invariant under translation by $n$-torsion points (since the vector bundles $\mathrm{W}_{n-1, n}$ are so, see e.g. [14, (14)]). Therefore, since the support of $D_{y, n}$ is contained in $E_{y, n}$, it must be equal to $E_{y, n}$. Hence $c_{1}\left(D_{y, n}\right)$ should be a multiple of $n^{2 g} \underline{\theta}$. On the other hand, (5.7) yields that

$$
c_{1}\left(D_{y, n}\right)=n^{g+2}(n-1)^{g-1}(n+1)^{g-1} \underline{\theta} .
$$

This is a contradiction as soon as $g \geq 3$. Hence, for $g \geq 3$, if the rank of $\Theta_{y}(n)$ attains the maximum for some $y \in A$ then the polarization must be reducible.

Finally we show the computation of (5.4) and (5.5). We use the Fourier-Mukai transform $\widehat{\Phi}_{\mathcal{P}}: D(A) \rightarrow D(\widehat{A})$ already invoked in $\$ 4$, and also the transform in the opposite direction $\Phi_{\mathcal{P}}: D(\widehat{A}) \rightarrow D(A)$, as well as their versions at the level of Chow rings modulo numerical equivalence $\widehat{\Phi}_{C H}: \mathcal{A}(A) \rightarrow \mathcal{A}(\widehat{A})$ and $\Phi_{C H}: \mathcal{A}(\widehat{A}) \rightarrow \mathcal{A}(A)$ (see [13, Proposition 1.21]). By GRR they commute with the Chern character. We use the following facts: (a) $\Phi_{\mathcal{P}} \circ \widehat{\Phi}_{\mathcal{P}}=(-1)_{A}^{*}[-g]$; (b) $\operatorname{ch}\left(\mathrm{W}_{a, b}\right)=a^{g} \exp \left(\frac{b}{a} \underline{\theta}\right)$ (this follows from (2.2) ); (c) $\Phi_{C H}\left(\exp \frac{b}{a} \underline{\theta}\right)=\left(\frac{b}{a}\right)^{g} \exp \left(-\frac{a}{b} \underline{\theta}\right)([14, \S 3.3])$; (d) If $G$ is symmetric then $\Phi_{\mathcal{P}}(F \otimes G)=\Phi_{\mathcal{P}}(F) \hat{*} \Phi_{\mathcal{P}}(G)[g]$ and $\widehat{\Phi}_{\mathcal{P}}(\mathcal{F} \hat{*} \mathcal{G})=\widehat{\Phi}_{\mathcal{P}}(\mathcal{F}) \otimes \widehat{\Phi}_{\mathcal{P}}(\mathcal{G})$ (this follows from [12, (3.7)] using that $\mathcal{F} \hat{*} \mathcal{G} \cong \mathcal{F} *(-1)^{*} \mathcal{G}$ ), where $*$ is the Pontryagin product). Therefore

$$
\begin{aligned}
& c h\left(\mathrm{~W}_{a, a+b} \hat{*} \mathrm{~W}_{b, a+b}\right) \stackrel{(d) \stackrel{(b)}{=}(c)}{=}(-1)^{g} \Phi_{C H}\left((a+b)^{g} \exp \left(-\frac{a}{a+b} \underline{\theta}\right)(a+b)^{g} \exp \left(-\frac{b}{a+b} \underline{\theta}\right)\right) \\
& =(a+b)^{2 g} \exp (\underline{\theta})
\end{aligned}
$$

This proves (5.4). Moreover

$$
\begin{aligned}
& c h\left(\left(\mathrm{~W}_{a, a+b} \otimes \mathrm{W}_{b, a+b}\right) \hat{*} \mathrm{~W}_{a, a+b}\right)= \\
\stackrel{(a)(b)(d)}{=} & (-1)^{g} \Phi_{C H}\left(\widehat{\Phi}_{C H}\left(a^{g} \exp \left(\frac{a+b}{a} \underline{\theta}\right) b^{g} \exp \left(\frac{a+b}{b} \underline{\theta}\right)\right) \cdot \widehat{\Phi}_{C H}\left(a^{g} \exp \left(\frac{a+b}{a} \underline{\theta}\right)\right)\right) \\
= & (-1)^{g} \Phi_{C H}\left(\widehat{\Phi}_{C H}\left((a b)^{g} \exp \left(\frac{(a+b)^{2}}{a b} \underline{\theta}\right)\right) \cdot \widehat{\Phi}_{C H}\left(a^{g} \exp \left(\frac{a+b}{a} \underline{\theta}\right)\right)\right) \\
\stackrel{(c)}{=} & (-1)^{g} \Phi_{C H}\left(\left((a b)^{g} \frac{(a+b)^{2 g}}{(a b)^{g}} \exp \left(-\frac{a b}{(a+b)^{2}} \underline{\theta}\right)\right) \cdot\left(a^{g} \frac{(a+b)^{g}}{(a)^{g}} \exp \left(-\frac{a}{a+b}\right)\right)\right) \\
= & \Phi_{C H}\left((a+b)^{3 g} \exp \left(-\frac{a(2 b+a)}{(a+b)^{2}} \underline{\theta}\right)\right) \\
\stackrel{(c)}{=} & (a+b)^{g} a^{g}(2 b+a)^{g} \exp \left(\frac{(a+b)^{2}}{a(2 b+a)} \underline{\theta}\right),
\end{aligned}
$$

where in the first equality we used that the vector bundles appearing in the calculation are symmetric, so that one can neglect the $(-1)_{A}^{*}$ in the formula $\Phi_{\mathcal{P}} \circ \widehat{\Phi}_{\mathcal{P}}=(-1)^{*}[-g]$. This proves (5.5).

Proof for $\mathbf{g}=\mathbf{2}$. In this case the irreducibility means that $\Theta$ is a smooth irreducible curve of genus 2 , and $A$ is its Jacobian. Assuming this, we claim that the isogeny $n_{A}$ restricted to any translate $\Theta_{y}$ is birational onto its image. We postpone this for the moment (see below), and we 
proceed with the proof. We note that the $n$-torsion points in $\Theta_{y}$ (if any) map all to 0 , which is therefore a point of multiplicity $\Theta_{y}(n)$ of the curve $n_{A, y}\left(\Theta_{y}\right)$. The class of the curve $n_{A}\left(\Theta_{y}\right)$ is $n^{2} \underline{\theta}$ (indeed $n_{A}^{*}\left(n_{A}\left(\Theta_{y}\right)\right)$ is the divisor $E_{y, n}$ of (5.1), whose class is $\left.n^{4} \underline{\theta}\right)$. Hence

$$
m(A, \Theta, 0):=\inf _{0 \in C \subset A}\left\{\frac{\Theta \cdot C}{\operatorname{mult}_{0} C}\right\} \leq \frac{2 n^{2}}{\Theta_{y}(n)},
$$

the infimum being taken over all reduced irreducible curves $C$ in $A$ passing through 0 . But $m(A, \Theta, 0)$ is the Seshadri constant of $\Theta$ at the point 0 (actually it is constant on all points of $A$, see [9, §5.1]), and it is known that, for irreducible principally polarized abelian surfaces $A$, $m(A, \Theta, 0)=\frac{4}{3}$ (as a particular case of a more general result concerning jacobians of hyperelliptic curves, see [5, Theorem 7], where Debarre attributes it to Lazarsfeld). This proves that, if $g=2$ and $\Theta$ is irreducible, $\Theta_{y}(n) \leq \frac{3}{2} n^{2}<2 n^{2}-1=n^{4}-\left(n^{2}-1\right)^{2}$. This proves the desired bound for $g=2$.

Finally, for the reader's convenience, we provide a proof of the previous claim, which is however a well known fact. We write $n_{A, y}:=\left(n_{A}\right)_{\mid \Theta_{y}}: \Theta_{y} \rightarrow A$. Let $x \in \Theta_{y}$. The fiber of $n_{A, y}$ at $x$ is, set theoretically, a subset of $\Theta_{y}$ of the form $\left\{x+\eta_{1}, x+\eta_{2}, \ldots, x+\eta_{k(x)}\right\}$, with $\eta_{i} \in A[n]$ and $\eta_{1}=0$. Hence $x \in \cap_{i=1}^{k(x)} \Theta_{y+\eta_{i}}$. In conclusion, the points $x$ such that $k(x)>1$ belong to the set of singular points of the effective divisor $E_{y, n}$ of (5.1), which is finite since $\Theta$ is irreducible. This proves what was claimed.

In conclusion for all $g \geq 2$, and $n \geq 2$, if there is a $y \in A$ such that the translate $\Theta_{y}$ contains the maximal number of $n$-torsion points, namely $n^{2 g}-\left(n^{2}-1\right)^{g}$, then $\Theta$ must be reducible. Therefore, by the decomposition theorem for p.p.a.v.'s ([4, Theorem 4.3.1]), $A$ splits as the polarized product of irreducible p.p.a.v.'s $\left(A_{i}, \underline{\theta}_{i}\right)$ for $i=1, \ldots, k$, of dimension $g_{i}$, with $g=\sum_{i=1}^{k} g_{i}$. Furthermore $\Theta_{y}=\sum_{i=1}^{k} p_{i}^{*} \Theta_{i, y_{i}}$, where $p_{i}$ denotes the projection $A \rightarrow A_{i}$, and it follows that, for all $i$, the translates $\Theta_{i, y_{i}}$ contain the maximal number of $n$-torsion points. Therefore $g_{i}=1$ for all $i$, since otherwise some of $\Theta_{i}$ 's would be reducible. It follows also that, for all $i, y_{i} \in A_{i}[n]$. This concludes the proof of Theorem $\mathrm{C}$.

\section{ACKLOWLEDGEMENTS}

The author thanks: Dragos Oprea for pointing out a gap in an earlier draft of this paper, Mihnea Popa for many conversations about semihomogeneous vector bundles a long time ago, Riccardo Salvati Manni for his encouragement and many discussions and suggestions, and the referee for very accurate remarks.

\section{REFERENCES}

[1] R.Auffarth, G. Codogni, Theta divisors whose Gauss map has a fiber of positive dimension, J. of Algebra 548 (2020), 153-161.

[2] R. Auffarth, G. P. Pirola, R. Salvati Manni, Torsion points on theta divisors, Proc. Amer. Math. Soc. 145 (2017), 89-99.

[3] M. Atiyiah, Vector bundles over an elliptic curve, Proc. Lond. Math. Soc. VII (1957), 414-452.

[4] C. Birkenhake and H. Lange, Complex abelian varieties, 2nd ed., Grundlehren der Mathematischen Wissenschaften 302, Springer, Berlin, 2004.

[5] O. Debarre, Seshadri constants of abelian varieties, in The Fano Conference, 379-394, Univ. Torino, Turin, 2004. 
[6] Z. Jiang, G. Pareschi, Cohomological rank functions on abelian varieties, Ann. Scient. Éc. Norm. Sup. 53 (2020), 815-846.

[7] G. Kempf, Complex Abelian Varieties and Theta Functions, Springer 1991.

[8] G. Kempf, Multiplication Over Abelian Varieties. Amer. J. of Math. 110 (1988), 765-773.

[9] R. Lazarsfeld, Positivity in Algebraic Geometry I, Springer-Verlag, 2004.

[10] V. Marcucci, G. Pirola, Points of order 2 on theta divisors, Atti Accad. Naz. Lincei Cl. Sci. Fis. Mat. Natur. Rend. Lincei Mat. Appl. 23 (2012), 319-323.

[11] S. Mukai, Semi-homogeneous vector bundles on an Abelian variety, J. Math. Kyoto Univ. 18 (1978), $239-272$.

[12] S. Mukai, Duality between $D(X)$ and $D(\widehat{X})$ with its application to Picard sheaves, Nagoya Math. J. 81 (1981), 153-175.

[13] S. Mukai, Fourier functor and its application to the moduli of bundles on an abelian variety, in Algebraic Geometry, Sendai 1985, 515-550, Adv. Stud. Pure Math., 10, North-Holland, 1987.

[14] D. Oprea, The Verlinde bundles and the semihomogeneous Wirtinger duality, J. Reine Angew. Math. 654 (2011), 181-217.

[15] G. Pareschi, Syzygies of abelian varieties, J. of the Amer. Math. Soc. 13 (2000), 651-664.

[16] G. Pareschi, M. Popa, Regularity on abelian variety, I, J. of the Amer. Math. Soc. 16 (2003), $285-302$.

[17] G. Pareschi, M. Popa, Regularity on Abelian varieties III: relationship with generic vanishing and applications, in Grassmannians, Moduli Spaces and Vector Bundles, pp. 141-167, AMS, 2011.

[18] G. Pareschi, R. Salvati Manni, 2-torsion points on theta divisors, International Mathematics Research Notices, rnz282, https://doi.org/10.1093/imrn/rnz282.

Dipartimento di Matematica, Università di Roma, Tor Vergata, Italy

Email address: pareschi@mat.uniroma2.it 